\title{
ORBITAL CONFIGURATIONS FOR GAS IN ELLIPTICAL GALAXIES
}

\author{
DAVID MERRITT \\ National Radio Astronomy Observatory, ${ }^{1}$ Charlottesville, Virginia \\ AND \\ TIM DE ZEEUW \\ Sterrewacht, Huygens Laboratorium, Leiden, The Netherlands \\ Received 1982 October 8; accepted 1982 December 2
}

\begin{abstract}
The complete set of simple closed orbits in triaxial potentials is presented. We show that quasi-stable configurations for gas in nonrotating elliptical galaxies are more numerous than previously suggested, so that the observed orientation of a dust lane is not related in any simple way to a galaxy's intrinsic shape. However, measurements of the direction of rotation of both the stars and gas in rotating dust lane galaxies may show whether elliptical galaxies are generically axially symmetric or triaxial.
\end{abstract}

Subject headings: galaxies: evolution — galaxies: structure

\section{INTRODUCTION}

Bertola and Galletta (1978) were the first to point out the existence of a class of elliptical galaxies containing a dust lane across the minor axis. The prototype of this class is the nearby galaxy NGC 5128 (Cen A). Since then the number of elliptical galaxies known to have gaseous disks has increased rapidly; the most recent optical survey (Hawarden et al. 1981) identifies 40 such galaxies, roughly half of which have obscuring lanes along the minor axis, and half along the major axis. Gunn (1979) showed that cold gas falling into an elliptical galaxy would tend to settle into a symmetry plane of the potential, and this argument was used by Tohline, Simonson, and Caldwell (1982) to derive the intrinsic shapes of a few dust lane elliptical galaxies, assuming that they were axially symmetric. Van Albada, Kotanyi, and Schwarzschild (1982, hereafter vAKS) extended these arguments by showing that, even in a rotating triaxial galaxy, there sometimes exists a continuous family of simple closed orbits on which gas might be trapped, not necessarily in a single plane, as it spirals into the center of the galaxy.

The rather widespread occurrence of dust lane elliptical galaxies and the generally smooth, regular appearance of the dust lanes themselves suggest that many of these galaxies are normal elliptical galaxies in which infalling gas has been trapped on closed orbits. It is therefore of interest to consider the complete set of simple closed orbits that might exist in a slowly rotating, triaxial galaxy. Two recent studies address this question.

\footnotetext{
1'The National Radio Astronomy Observatory is operated by Associated Universities, Inc., under contract with the National Science Foundation.
}

De Zeeuw and Merritt (1983) used analytic and numerical methods to study orbits in the plane of rotation of an elliptical galaxy. Magnenat (1982) and Heisler, Merritt, and Schwarzschild (1982, hereafter HMS) analyzed in detail the behavior of the tilted family of orbits described by vAKS.

The purpose of the present Letter is to summarize the results of these various investigations and to elucidate the relation between the appearance of a dust lane and the basic properties (shape, rotation) of the underlying galaxy. We show that it is probably impossible to determine the intrinsic shape of an elliptical galaxy from the observed orientation of its dust lane. However, measurements of the rotation curves of both the stars and the gas in a large number of dust lane elliptical galaxies can help determine whether elliptical galaxies as a class are axially symmetric, or triaxial.

\section{FAMILIES OF ORBITS}

\section{a) Nonrotating Galaxies}

Consider a nonrotating elliptical galaxy, in which the density is constant on similar, roughly ellipsoidal surfaces. If the galaxy is rotationally symmetric about any axis, then closed circular orbits will exist about that axis at all energies. (For specific potentials, e.g., Keplerian ones, noncircular closed orbits may also exist.) Gas or dust that falls into the galaxy can settle onto these orbits on a time scale determined by dissipative processes (Tohline, Simonson, and Caldwell 1982). If the galaxy is oblate, gas in such an orbit will circle the short axis, so that in projection the galaxy will appear to be bisected by an obscuring lane that runs along the 
ivi major axis (e.g., NGC 3283). A galaxy with this ap$\therefore$ pearance will be called "type L." If the galaxy is prolate, the absorbing lane will appear to lie along the minor axis, giving rise to a type $\mathrm{S}$ galaxy (e.g., NGC 5363). As pointed out by Tohline and others, these arguments can be turned around to derive the intrinsic shapes of nonrotating elliptical galaxies from the appearance of their obscuring lanes, assuming that the galaxies are axially symmetric.

It is easy to show, however, that more families of simple closed orbits must exist. Consider two test particles that oscillate in linear orbits along the long and short axes of an ellipsoidal galaxy. The oscillation period will be shortest for the particle moving along the short axis, since the potential gradient is greatest in that direction. As its energy is increased, the short axis particle spends more and more of its time out of the harmonic core, and its period goes up. At some energy the period of the finite-amplitude, short axis orbit will become equal to that of the long axis orbit in the core, and a second, highly elongated orbit will branch off ("bifurcate") from the short axis orbit. Both this "resonant" orbit and the axial orbit will exist at all higher energies, although one or both may be unstable.

De Zeeuw and Merritt (1983) derived approximate analytic expressions for the energies of appearance of the resonant orbits in a general triaxial galaxy and checked them against orbits calculated numerically in Schwarzschild's (1979) model potential. Using the formulae in that paper, we find that the radius $R_{b}$ of the shortest axial orbit which is unstable to bifurcation in a given principal plane can be written approximately as

$$
\left(R_{b} / R_{c}\right)^{2}=f(p, q)\left[1-\left(\kappa_{1} / \kappa_{2}\right)\right] \text {. }
$$

$R_{c}$ is the core radius of the galaxy density distribution measured along the long axis of the resonant orbit; $\kappa_{1}$ and $\kappa_{2}$ are the harmonic (core) oscillation frequencies along the long and short axes lying in the principal plane; and $f$ is a function of the two axial ratios $(p, q)$ of the three-dimensional density distribution. Note that $R_{b} / R_{c}$ does not depend on the radial form of the galactic density profile.

In the equatorial plane of an axisymmetric galaxy, $\kappa_{1}=\kappa_{2}$, and equation (1) says that resonant orbits (circular in this case) exist at all radii. But resonant orbits also exist in any orthogonal plane. Figure 1 gives $R_{b} / R_{c}$ as a function of galaxy ellipticity in oblate and prolate galaxies, for orbits lying perpendicular to the equatorial plane, i.e., "polar" orbits. Even in very flattened galaxies, this family of resonant orbits extends inward to one or two core radii and outward to infinity. This is illustrated for orbits in a specific potential in Figure $2 a$. Note that these orbits are nearly circular except near bifurcation.

Gas falling into an elliptical galaxy can settle onto any family of orbits which satisfies two conditions: the

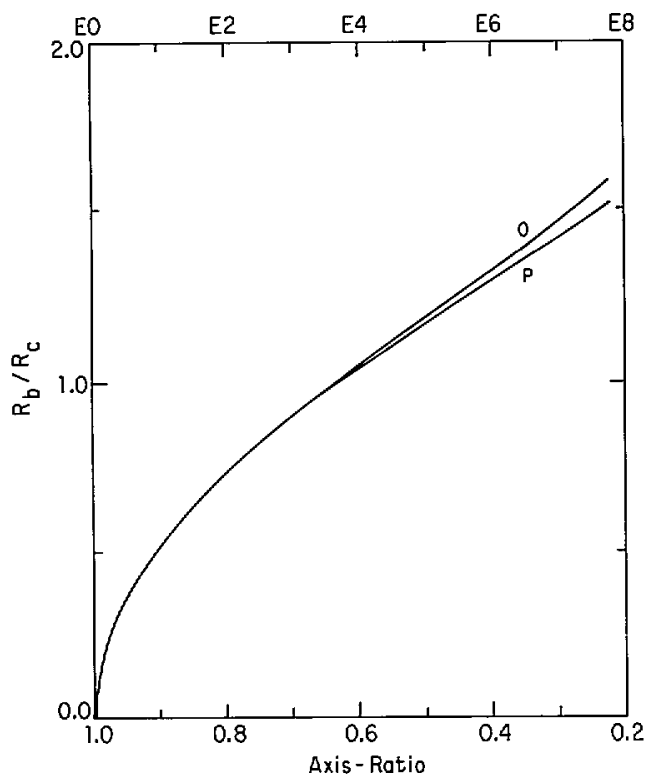

FIG. 1.-Bifurcation radius as a function of galaxy ellipticity in nonrotating prolate and oblate spheroids.

orbits must be non-self-intersecting, so that the orbital motion is not immediately dissipated by collisions; and they must be stable, in the sense that orbits near in space to any closed one must precess about it. If the latter condition is not satisfied, any misalignment of the orbiting gas from the principal plane will grow with time, due to the combined effects of differential precession and dissipation. All the orbits discussed here satisfy the first condition, except at very low energies where they bifurcate from the axial orbit; the bifurcation radius defines the point at which infalling gas would quickly lose its ordered motion and fall into the galaxy center. The second condition is not satisfied for polar orbits in strictly axisymmetric galaxies, however, since a small misalignment causes these to precess about the polar axis and settle into the equatorial plane (Tohline, Simonson, and Caldwell 1982).

But the precession time for gas in a large-radius orbit can be very long, so that polar orbits might exist for a significant fraction of a Hubble time. The best evidence for this is the large number of disk galaxies which are observed to be circled by rings of gas over the pole (Schechter 1982); the "Spindle" galaxy, NGC 2685, is the most famous example (cf. Shane 1980).

The instability of polar orbits can also be removed by making the underlying galaxy triaxial. Heiligman and Schwarzschild (1979) have shown that resonant orbits in two of the three principal planes of a triaxial galaxy are generally stable; only the orbit circling the intermediate axis is unstable. These two stable families may be considered as analogs to the equatorial and polar orbit families in a pure axisymmetric galaxy. 
(a)
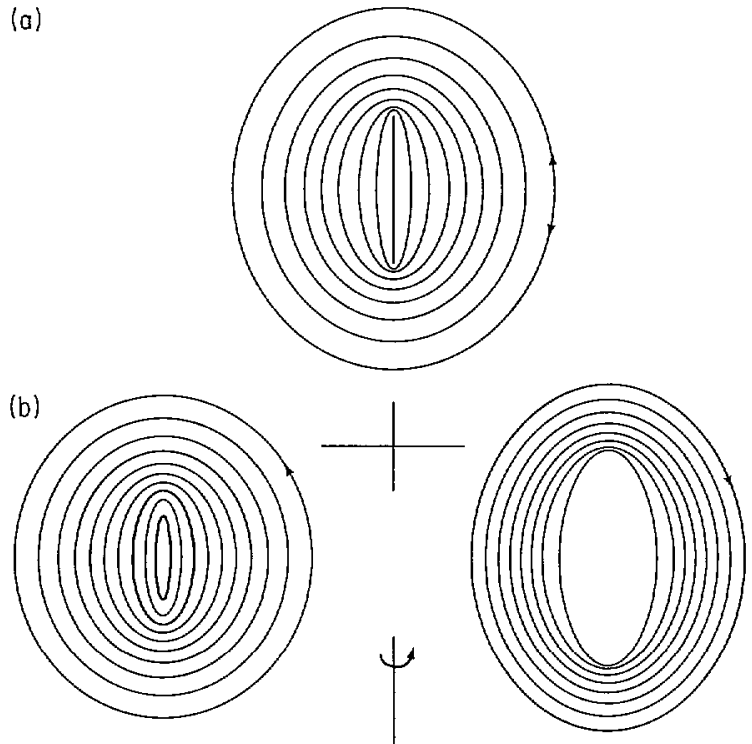

(c)

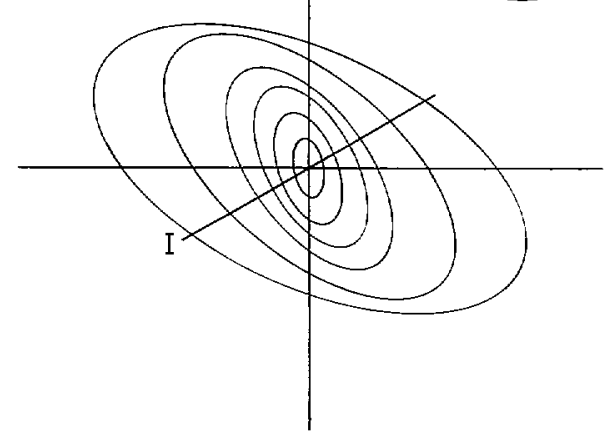

FIG. 2.- (a) Resonant orbits in a principal plane of Schwarzschild's (1979) triaxial galaxy; the axis ratio of the density distribution in the plane is $1: 1.6$. Core radii along the two principal axes are indicated by the cross. (b) Resonant orbits in the same galaxy, now rotating slowly about an axis perpendicular to the orbital plane; the retrograde (left) and direct (right) families have slightly different shapes. (c) Anomalous orbits in the rotating galaxy. At small radii these orbits lie in the principal plane containing the rotation axis, while at large radii they merge with the resonant orbits in the plane of rotation. Tilt occurs about the intermediate axis, labeled "I."

It follows that an obscuring lane that lies along the apparent major axis of an elliptical galaxy (type L) may be produced either by matter moving in a circular orbit in the equatorial plane of an oblate galaxy, or by matter in a resonant orbit passing through the symmetry axis of a prolate galaxy, or by matter in a resonant orbit circling the short axis of a triaxial galaxy. Similarly, type S galaxies may be oblate, prolate, or triaxial. There is no one-to-one correspondence between the appearance of a dust lane and the intrinsic shape of the galaxy.

\section{b) Rotating Galaxies}

Consider next galaxies in which the figure is allowed to rotate. Rotation about a symmetry axis is trivial, since it produces no change in the potential. The next simplest case is rotation of an oblate or prolate spheroid about an axis in the equatorial plane, or of a triaxial ellipsoid about any principal axis. "Tumbling" motion of this sort has been shown to be possible in prolate or nearly prolate models (e.g., Schwarzschild 1982), but so far no model has been produced of a tumbling oblate spheroid.

Figure rotation has two effects. (1) Orbits in the plane of rotation take on slightly different shapes depending on whether they are direct or retrograde with respect to the figure (De Zeeuw and Merritt 1983). The direct family terminates at $R \approx R_{b}$, just as in the nonrotating case; the retrograde family continues inward, but becomes very thin (Fig. 2b). (2) One of these families-either the retrograde, if figure rotation is about the short axis, or the direct, if rotation is about the long axis-is unstable to perturbations out of the plane of rotation over a wide radial range (Binney 1981). In both cases, however, an additional stable family of orbits (called "anomalous" by vAKS) exists that is inclined to the plane of rotation; the tilt is about the intermediate figure axis and increases inward until the orbit lies in the principal plane containing the axis of rotation (vAKS; HMS; Magnenat 1982; Durisen et al. 1983; Fig. 2c). Gas falling in along the unstable family would probably "flip up" into the anomalous family and end up in a principal plane orthogonal to the plane of rotation.

If we restrict our attention to gas disks in the inner few kiloparsecs of slowly rotating galaxies, the relevant families are the resonant orbits in the plane of rotation, and the anomalous orbits are perpendicular to them. Just as in the nonrotating case, then, there are two principal planes into which gas would be expected to settle.

\section{OBSERVATIONAL CONSEQUENCES}

In Table 1 the possible orbital configurations of gas in a rotating triaxial galaxy are shown schematically, under the following assumptions: $(a)$ the direction of rotation of the stars matches that of the figure; $(b)$ the mass distribution is similar to the light distribution; $(c)$ the figure rotates about either the long or the short axis; intermediate axis rotation seems unlikely, but has not yet been ruled out; and $(d)$ the gas disk does not extend beyond the region, typically many kiloparsecs, where the anomalous family is strongly tilted with respect to all of the coordinate axes (as in the model of vAKS for NGC 5128). The third column gives the general appearance of the galaxy as seen from the two principal axes lying in the plane of the dust lane; the tilted extensions of the anomalous family at large radii are indicated by dashed lines. The fourth column gives the directions of the angular momentum vectors of stars and gas respectively; rotation in the plane of the sky is indicated by a simple 
TABLE 1

Orbital Configurations of Gas in a Rotating Triaxial Galaxy

\begin{tabular}{|c|c|c|c|c|}
\hline ROTATION AXIS & ORBIT & APPEARANCE & MOMENTA & TYPE \\
\hline Short & Resonant & & $t, t$ & L \\
\hline Short & Anomalous & & $\uparrow, \rightarrow$ & S \\
\hline Long & Resonant & & $4, t$ & S \\
\hline Long & Anomalous & & $\uparrow,-$ & L \\
\hline & & & $1,-$ & \\
\hline
\end{tabular}

line. The fifth column gives the galaxy type, either $\mathrm{L}$ or $\mathrm{S}$, according to the scheme described above.

The fact that the number of orbital configurations is so limited in the rotating triaxial case suggests the following test for triaxiality. If dust lane elliptical galaxies should turn out to always be described by one of the four basic types of Table 1, based both on the orientation of their dust lanes and the angular momentum vectors of stars and gas, then it would be reasonable to conclude that elliptical galaxies as a class are triaxial and not axially symmetric. If, however, exceptions are observed, then at least some galaxies would have to be very nearly axisymmetric. For example, a type $\mathrm{L}$ galaxy with a retrograde dust lane could not be triaxial because the corresponding resonant orbit would be unstable to perturbations out of the plane, as described above. For the same reason, a triaxial type $S$ galaxy could not support gas in a direct orbit.

The test can be made even simpler if it is assumed, as recent observations seem to suggest (Schechter and Gunn 1979), that elliptical galaxies always rotate about their short axis. In this case the only possible configurations for gas in triaxial galaxies are the first two shown in Table 1. Then, under the triaxial hypothesis, type $L$ galaxies should always have direct dust lanes, while in type $\mathbf{S}$ galaxies the gas should rotate orthogonally to the stars at small radii and retrograde at larger radii. Unfortunately, these two configurations are also the only ones allowed by a purely prolate tumbling galaxy; however, the good correlation observed between rotation and flattening for the fainter elliptical galaxies suggests that these at least are nearly oblate (Davies et al. 1983), so the hypothesis that most galaxies are tumbling prolate bars can probably be rejected outright.

Observations of the stars and gas in four dust lane elliptical galaxies were recently made by Sharples et al. (1983). In two cases (NGC 5363 and UKS 0151-496), rotation curves were obtained for both the stars and the gas, the latter from emission-line regions in the dust lanes. Both galaxies are type $\mathrm{S}$, and in both the angular momentum vectors of stars and gas are orthogonal. This is the second configuration shown in Table 1 and suggests that both galaxies are rotating about a short axis, while the dust lanes follow anomalous orbits. Although these observations are consistent with the triaxial hypothesis, they do not confirm it, since both galaxies could equally well be prolate. For this reason, more observations of dust lane galaxies would be desirable: either of type $\mathrm{L}$ galaxies, or of type $\mathrm{S}$ galaxies in which the stars rotate about the apparent major axis (if such galaxies exist).

It should also be emphasized that some $L$ type galaxies could be early-type spirals masquerading as dust lane ellipticals. Stars and gas in these systems probably have a common origin and would always be rotating in the same direction.

Nevertheless, we believe that further observations of dust lane galaxies would be of great help in determining the intrinsic shapes of elliptical galaxies.

The authors are indebted to the anonymous referee whose comments greatly improved the presentation of these results. 


\section{REFERENCES}

Bertola, F., and Galletta, G. 1978, Ap.J. (Letters), 226, L115.

Binney, J. 1981, M.N.R.A.S., 196, 455.

Davies, R. I., Efstathiou, G., Fall, S. M., Illingworth, G., and Iol Schechter, P. L. 1983, Ap.J., 266, 41.

int de Zeeuw, P. T., and Merritt, D. R. 1983, Ap. J., 267, in press.

Durisen, R. H., Tohline, J. E., Burns, J. A., and Dobrovolskis, A. R. 1983, Ap.J., 264, 392.

Gunn, J. E. 1979, in Active Galactic Nuclei, ed. C. Hazard and S. Mitton (Cambridge: Cambridge University Press), p. 213.

Hawarden, T. G., Elson, R. A. W., Longmore, A. J., Tritton, S. B., and Corwin, H. G. 1981, M.N.R.A.S., 196, 747.

Heiligman, G., and Schwarzschild, M. 1979, Ap.J., 233, 872

Heisler, J., Merritt, D., and Schwarzschild, M. 1982, Ap. J., 258 490 (HMS).

Tim DE ZEEUw: Sterrewacht Leiden, Postbox 9513, 2300 RA Leiden, The Netherlands

David Merritr: National Radio Astronomy Observatory, Edgemont Road, Charlottesville, VA 22901
Magnenat, P. 1982, Astr. Ap., 108, 89.

Schechter, P. L. 1982, private communication.

Schechter, P. L., and Gunn, J. E. 1979, $A p . J ., 229,472$.

Schwarzschild, M. 1979, Ap.J., 232, 236.

Schwarzschild, M. 1982, Ap. J., $263,599$.

Shane, W. W. 1980, Astr. Ap., 82, 314.

Sharples, P. M., Carter, D., Hawarden, T. G., and Longmore, A. J. 1983, M.N.R.A.S., submitted.

Tohline, J. E., Simonson, G. F., and Caldwell, N. 1982, Ap. J., 252, 92.

van Albada, T. S., Kotanyi, C. G., and Schwarzschild, M. 1982, M.N.R.A.S., 198, 303 (vAKS). 
